\title{
Segmentation of the Globus Pallidus Internus Using Probabilistic Diffusion Tractography for Deep Brain Stimulation Targeting in Parkinson Disease
}

\author{
(DE.H. Middlebrooks, (DI.S. Tuna, DS.S. Grewal, (D). Almeida, DM.G. Heckman, DE.R. Lesser, DK.D. Foote, DM.S. Okun, and
}

(D) V.M. Holanda

\begin{abstract}
BACKGROUND AND PURPOSE: Although globus pallidus internus deep brain stimulation is a widely accepted treatment for Parkinson disease, there is persistent variability in outcomes that is not yet fully understood. In this pilot study, we aimed to investigate the potential role of globus pallidus internus segmentation using probabilistic tractography as a supplement to traditional targeting methods.
\end{abstract}

MATERIALS AND METHODS: Eleven patients undergoing globus pallidus internus deep brain stimulation were included in this retrospective analysis. Using multidirection diffusion-weighted MR imaging, we performed probabilistic tractography at all individual globus pallidus internus voxels. Each globus pallidus internus voxel was then assigned to the $1 \mathrm{ROI}$ with the greatest number of propagated paths. On the basis of deep brain stimulation programming settings, the volume of tissue activated was generated for each patient using a finite element method solution. For each patient, the volume of tissue activated within each of the 10 segmented globus pallidus internus regions was calculated and examined for association with a change in the Unified Parkinson Disease Rating Scale, Part III score before and after treatment.

RESULTS: Increasing volume of tissue activated was most strongly correlated with a change in the Unified Parkinson Disease Rating Scale, Part III score for the primary motor region (Spearman $r=0.74, P=.010$ ), followed by the supplementary motor area/premotor cortex (Spearman $r=0.47, P=.15)$.

CONCLUSIONS: In this pilot study, we assessed a novel method of segmentation of the globus pallidus internus based on probabilistic tractography as a supplement to traditional targeting methods. Our results suggest that our method may be an independent predictor of deep brain stimulation outcome, and evaluation of a larger cohort or prospective study is warranted to validate these findings.

ABBREVIATIONS: DBS = deep brain stimulation; $\mathrm{FGATIR}=$ fast gray matter acquisition $\mathrm{T}$ inversion recovery; $\mathrm{GPe}=$ globus pallidus externus; $\mathrm{GPi}=\mathrm{globus}$ pallidus internus; $\mathrm{Ml}=$ primary motor cortex; $\mathrm{MNI}=$ Montreal Neurological Institute; $\mathrm{PD}=$ Parkinson disease; $\mathrm{SMA} / \mathrm{PMC}=$ supplementary motor area/premotor cortex; $\mathrm{SN}=$ substantia nigra; STN = subthalamic nucleus; UPDRS III = Unified Parkinson Disease Rating Scale, Part III; VTA = volume of tissue activated

G lobus pallidus internus (GPi) deep brain stimulation (DBS) is an established treatment for Parkinson disease (PD). ${ }^{1}$ Successful motor symptom reduction has been validated in randomized clinical trials with DBS of the GPi. ${ }^{2,3}$ Similar to other conditions and other targets, variability in outcomes remains, and the underpinning of such variability remains incompletely understood. While there are numerous potential mechanistic bases for

Received December 17, 2017; accepted after revision February 24, 2018.

From the Departments of Radiology (E.H.M.) and Neurosurgery (S.S.G.) and Division of Biomedical Statistics and Informatics (M.G.H., E.R.L.), Mayo Clinic, Jacksonville, Florida; Departments of Radiology (I.S.T.), Neurology (L.A., M.S.O.), and Neurosurgery (K.D.F.), University of Florida, Gainesville, Florida; and Center of Neurology and Neurosurgery Associates (V.M.H.), BP-A Beneficência Portuguesa de São Paulo, São Paulo, Brazil.

Please address correspondence to Erik H. Middlebrooks, MD, 4500 San Pablo Rd, Jacksonville, FL 32224; e-mail: Middlebrooks.Erik@mayo.edu

$\checkmark$ Indicates article with supplemental on-line photo.

http://dx.doi.org/10.3174/ajnr.A5641 these outcomes, 1 potential explanation lies in the limitations of preoperative functional targeting.

Traditional methods of indirect targeting, whereby DBS-lead targets are defined by a rigid coordinate-based stereotaxy using readily identifiable macrostructures (eg, coordinate-based localization relative to a line through the anterior/posterior commissure line), have become outdated as stand-alone targeting methods. The use of more sophisticated direct targeting has become a standard for GPi-DBS lead implantation, using MR imaging sequences such as fast gray matter acquisition $\mathrm{T} 1$ inversion recovery $\left(\right.$ FGATIR) ${ }^{4}$ and diffusion tensor imaging tractography, ${ }^{5,6}$ as well as software advancements in targeting (eg, high-quality deformable brain atlases). ${ }^{4}$ Intraoperatively, the functional targeting is often supplemented by careful microelectrode recording and macrostimulation, fine-tuning the final position of the DBS electrode before the lead is secured in place. Nevertheless, preoperative targeting and subsequent DBS programming based on image- 
guided functional information are not routinely performed at many institutions worldwide and are being recognized by multiple groups as a potential strategy for surgical planning., ${ }^{7,8}$

With continued advances in diffusion-weighted MR imaging during the preceding decade, it has become possible to routinely obtain high-quality multidirection diffusion imaging in the clinical setting. Diffusion-based probabilistic tractography has previously been used in the segmentation of various deep nuclei based on structural-connectivity profiles, particularly within the thalamus. ${ }^{9-12}$ A previous study examining such MR imaging probabilistic tractography to segment the globus pallidus internus in healthy controls found mixed results with multiple datasets and failed to demonstrate some known corticopallidal connections. ${ }^{13}$

In the current pilot study, we examined the role of segmentation of the globus pallidus internus based on probabilistic tractography in the setting of deep brain stimulation. To our knowledge, there are no prior studies evaluating globus pallidus segmentation on the basis of probabilistic tractography in patients with GPi-DBS. On the basis of our experience with DBS localization in the GPi, we believe that the optimal GPi target for control of movement symptoms lies within the posterior ventral GPi. Anatomic and physiologic studies in primates and humans have also shown that the sensorimotor territory of the GPi is ventral and posterior. ${ }^{14,15}$ Thus, we hypothesized that clinical improvement will correlate with the volume of tissue activated (VTA) overlapping the GPi regions most connected to the primary and supplementary motor areas.

\section{MATERIALS AND METHODS}

The use of the study data was approved by the University of Florida institutional review board. The study design was a retrospective analysis of consecutive patients with the diagnosis of PD undergoing GPi-DBS who had undergone multidirection diffusion-weighted MR imaging as part of their preoperative targeting MR imaging. Seventeen such patients were identified. Six patients were excluded from further analysis (1 patient had bilateral DBS leads placed with no Unified Parkinson Disease Rating Scale, Part III [UPDRS III] score recorded between lead placements, 1 patient did not have the needed raw diffusion data archived, 2 patients had inadequate postoperative imaging for lead localization, and 2 patients had excessive head motion). Eleven patients underwent further analysis. Three of the 11 patients underwent bilateral lead placement at 2 separate time points. For these 3 patients, only the information obtained from the first lead placement was used in this study to satisfy the statistical assumption of independent measurements.

\section{Chart Review}

A retrospective chart review was conducted for the collection of relevant clinical and demographic data. Demographic and disease information collected included age at DBS, sex, disease duration, and handedness. Each patient's most effective stimulation settings were also recorded. Settings were initially determined to maximize symptom control and minimize stimulation-induced adverse effects. The most common adverse effects were pulling of the contralateral arm and/or leg due to stimulation of the posterior limb of the internal capsule when the deeper contacts were used in some patients. The UPDRS III score was measured at the preoperative visit and the 6-month follow-up; change in the UPDRS III score (preoperative minus 6-month follow-up) was calculated and was the primary outcome measure of the study. Tremor-suppressing medications were held stable at the preoperative optimized dose for the duration of the study.

\section{Image Acquisition}

All patients underwent preoperative MR imaging for DBS planning. As part of the imaging protocol, patients underwent a T1weighted MPRAGE and multidirection diffusion-weighted imaging. All scans were obtained on a 3T Verio scanner (Siemens, Erlangen, Germany) using a 12-channel head coil.

The multidirection diffusion-weighted imaging was acquired at an isotropic resolution of $1.6 \times 1.6 \times 1.6 \mathrm{~mm}$ with no gap for a total of 70 slices. A total of 64 diffusion directions were acquired at a b-value of $1000 \mathrm{~s} / \mathrm{mm}^{2}$. Total imaging time was 12 minutes 14 seconds $(\mathrm{TR}=10,800 \mathrm{~ms}, \mathrm{TE}=100 \mathrm{~ms}$, phase partial Fourier $=$ $5 / 8$, generalized autocalibrating partially parallel acquisition $=2$, bandwidth $=1098 \mathrm{~Hz} / \mathrm{Px}$, and EPI factor $=130$ ).

The MPRAGE imaging was acquired in the axial plane after the administration of gadolinium-based intravenous contrast $(0.1 \mathrm{~mm} / \mathrm{kg})$. Imaging parameters included an in-plane resolution of $0.6 \times 0.6 \mathrm{~mm}$ with a slice thickness of $1.0 \mathrm{~mm}, \mathrm{TR}=1720$ $\mathrm{ms}, \mathrm{TE}=3.29 \mathrm{~ms}, \mathrm{TI}=865 \mathrm{~ms}$, flip angle $=9^{\circ}$, and bandwidth $=$ $170 \mathrm{~Hz} / \mathrm{Px}$. Image acceleration included a 7/8 phase partial Fourier imaging and generalized autocalibrating partially parallel acquisition $=2$.

Additionally, a volumetric FGATIR sequence was obtained, as previously reported, ${ }^{4}$ for targeting purposes.

All postoperative CT scans were obtained on an Aquilion scanner (Toshiba Medical Systems, Tokyo, Japan) using a $0.5 \times 0.5$ $\mathrm{mm}$ in-plane resolution with a slice thickness of $1 \mathrm{~mm}$.

\section{Surgical Procedure}

GPi-DBS was planned contralateral to the patient's most symptomatic upper extremity. All surgical procedures were performed by the same surgeon (K.D.F.). For targeting, brain MR imaging was acquired before the initial procedure. On the day of the operation, a Cosman-Roberts-Wells head ring was placed, and stereotactic high-resolution CT was performed. In-house software was used to fuse the CT and MR imaging data. The postcontrast T1-weighted MPRAGE and FGATIR MRI sequences were paired with a deformable brain atlas in order to identify the corpus striatum, the globus pallidus, and the optic tract anatomy. Single microelectrode recording was sequentially performed to establish the border between the globus pallidus externus (GPe) and the GPi by the sound of the border cells. The inferior GPi border was established by the sound of the optic tract. Macrostimulation was performed to assure the best lead position.

\section{ROI}

In contrast to the prior study in healthy controls, we limited our connectivity targets to known predominant connections of the globus pallidus internus. ${ }^{13}$ ROIs for probabilistic tractography were defined in Montreal Neurological Institute (MNI) template space and created using the FSLView "atlas" function (https://fsl. fmrib.ox.ac.uk/fsl/fslwiki/FslView). Masks were created for the 
caudate, GPe, GPi, putamen, and subthalamic nucleus (STN) from the MNI Basal Ganglia Human Area Template. ${ }^{16}$ The substantia nigra (SN) mask was generated using atlasing of the basal ganglia. ${ }^{17}$ Cortical ROIs were concatenated for the primary motor cortex (M1) and supplementary motor area and premotor cortex (SMA/PMC) from the MNI Human Motor Area Template. ${ }^{18}$ Masks for the prefrontal cortex and thalamus were generated from the Harvard-Oxford Cortical and Subcortical Atlases, respectively. ${ }^{19-22}$ Last, the pedunculopontine nucleus was manually drawn in MNI space to approximate the location of this poorly visualized structure within the midbrain-pontine tegmentum inferior, posterior, and lateral to the red nucleus.

\section{Imaging Preprocessing}

Diffusion data underwent standard preprocessing consisting of re-alignment and eddy current correction using the FMRIB Software Library (FSL; http://www.fmrib.ox.ac.uk/fsl). The diffusion-weighted data were normalized to the MNI_ICBM_2009b_ NLIN_ASYM atlas space (http://neuro.debian.net/pkgs/mniicbm152-nlin-2009c.html).

Preoperative MPRAGE and postoperative CT were coregistered by a 2-stage linear registration consisting of a rigid and subsequent affine registration used in the Advanced Normalization Tools software package (http://stnava.github.io/ANTs/). ${ }^{19}$ Next, these volumes were also normalized to the MNI_ICBM_2009b_ NLIN_ASYM atlas space with the SyN registration approach in the Advanced Normalization Tools. ${ }^{23,24}$

\section{Diffusion Data Processing}

Voxelwise diffusion parameters were estimated from the preprocessed diffusion data using a Markov Chain Monte Carlo sampling in the FSL BEDPOSTX (https://fsl.fmrib.ox.ac.uk/fsl/ fslwiki/FDT) function. Next, probabilistic tractography was performed with PROBTRACKX2 (https://fsl.fmrib.ox.ac.uk/fsl/ fslwiki/FDT) in FSL to calculate a probability of connection of each voxel within the left and right GPi masks to each of the 10 previously defined target masks. Five thousand total fiber tracks were generated with a curvature threshold $=0.2$, step length $=0.5$ $\mathrm{mm}$, and maximum steps $=2000$. Last, all GPi voxels were classified by the 1 target mask with the greatest number of propagated paths by the FSL "find_the_biggest" function.

\section{Volume of Tissue Activated Modeling}

The DBS electrodes were localized on the normalized postoperative CT using the Lead-DBS software package (http://www.leaddbs.org). ${ }^{25}$ Manual corrections, when necessary, were performed to ensure the accuracy of the contact locations. Once contact locations were determined, the VTA was simulated using the patient's most effective programming settings, as implemented in Horn et al. ${ }^{8}$ Briefly, a finite-element method solution was derived using a tissuespecific conductivity model. The volume of overlap between each patient's simulated VTA and each of the 10 segments within the patient-specific GPi segmentation was calculated.

\section{Statistical Analysis}

Continuous variables were summarized with the sample median and range. Categoric variables were summarized with the number and percentage of patients. The degree of association between the VTA in each of the 10 different brain regions and the change in the UPDRS III score from preoperative to 6-month follow-up visits were evaluated by estimating the Spearman correlation coefficient $r$. Although Spearman tests of correlation were also performed, due to the pilot nature of the study and the corresponding small sample size, results of these tests are primarily given for completeness, and emphasis should be placed on the Spearman correlation coefficient $r$ values when interpreting results. For brain regions in which $<3$ patients had a value greater than zero regarding the VTA, the Spearman correlation was not assessed because these regions were essentially noninformative due to lack of variability in measures. Associations of age and disease duration with change in the UPDRS III score were examined in a similar manner. $P$ values $\leq .05$ were considered statistically significant. All statistical tests were 2-sided. Statistical analyses were performed using SAS (Version 9.4; SAS Institute, Cary, North Carolina) and R Statistical and computing software (Version 3.2.3; http://www. r-project.org).

\section{RESULTS}

A summary of patient characteristics, DBS characteristics, and outcomes is shown in the Table. The median age at DBS was 62 years (range, 49-73 years), almost all patients were male $(n=10$ [91\%]), and the median disease duration was 9 years (range, $6-16$ years). The median UPDRS III score was 42 (range, 27-55) before the operation and 21 (range, 13-40) at 6-month follow-up, corresponding to a median decrease of 15 (range, 12-point increase to a 31-point decrease).

Group averages for each of the 10 segmented regions are shown in Fig 1. Of the 10 GPi segments assessed, only 4 (GPe, M1, SMA/PMC, SN) had at least some VTA in $>2$ patients (Table and On-line Figure) and were therefore evaluated further for association with change in the UPDRS III score. For these 4 GPi segments, the VTA was most strongly correlated with change in the UPDRS III score for the segment most connected to the M1 region (Spearman $r=0.74, P=.010$, Fig 2), followed by the SMA/ PMC (Spearman $r=0.47, P=.15$, Fig 2), GPe (Spearman $r=$ $0.16, P=.64$, Fig 2 ), and SN (Spearman $r=0.15, P=.67$, Fig 2) regions. Of note, we did not observe a strong correlation with change in the UPDRS III score for either age at DBS (Spearman $r$, $-0.23, P=.49$ ) or disease duration (Spearman $r=-0.08, P=$ .81 ), indicating that these 2 variables would have had minimal confounding influence on the correlations between the VTA and change in the UPDRS III score.

\section{DISCUSSION}

The GPi is considered the primary output structure of the basal ganglia and is known to have an increase in its neuronal activity before the onset of PD motor symptoms. ${ }^{26} \mathrm{GPi}$-DBS has been successfully used to abolish dyskinesias in patients with treatment-resistant hyperkinetic movements, tremor, and dystonia in PD. ${ }^{15,27}$ In this pilot study, we assessed a novel method of segmentation of the GPi based on probabilistic tractography. We found that a greater VTA in the somatosensory region of the GPi within our defined M1 segment and, to a lesser degree, the SMA/ 
Patient and deep brain stimulation characteristics and outcomes ${ }^{a}$

\begin{tabular}{|c|c|}
\hline Variable & $\begin{array}{c}\text { Summary } \\
(N=11)\end{array}$ \\
\hline \multicolumn{2}{|l|}{ Patient characteristics } \\
\hline Age at DBS (mean) (range) (yr) & $62(49-73)$ \\
\hline Male sex (No.) (\%) & $10(90.9 \%)$ \\
\hline Disease duration (mean) (range) (yr) & $9(6-16)$ \\
\hline Handedness (right) (No.) (\%) & $10(90.9 \%)$ \\
\hline \multicolumn{2}{|l|}{ Deep brain stimulation information } \\
\hline \multicolumn{2}{|l|}{ Volume of tissue activated $\left(\mathrm{mm}^{3}\right)$} \\
\hline \multicolumn{2}{|l|}{ Caudate } \\
\hline No. (\%) of patients with values of $>0$ & $0(0.0 \%)$ \\
\hline Median (minimum, maximum) & $0.0(0.0,0.0)$ \\
\hline \multicolumn{2}{|l|}{ GPe } \\
\hline No. (\%) of patients with values of $>0$ & $11(100.0 \%)$ \\
\hline Median (minimum, maximum) & $19.3(0.8,78.4)$ \\
\hline \multicolumn{2}{|l|}{$\mathrm{Ml}$} \\
\hline No. (\%) of patients with values of $>0$ & $8(72.7 \%)$ \\
\hline Median (minimum, maximum) & $3.6(0.0,42.9)$ \\
\hline \multicolumn{2}{|l|}{ PPN } \\
\hline No. (\%) of patients with values of $>0$ & $1(9.1 \%)$ \\
\hline Median (minimum, maximum) & $0.0(0.0,7.9)$ \\
\hline \multicolumn{2}{|l|}{ PFC } \\
\hline No. (\%) of patients with values of $>0$ & $2(18.2 \%)$ \\
\hline Median (minimum, maximum) & $0.0(0.0,5.5)$ \\
\hline \multicolumn{2}{|l|}{ Putamen } \\
\hline No. (\%) of patients with values of $>0$ & $2(18.2 \%)$ \\
\hline Median (minimum, maximum) & $0.0(0.0,38.8)$ \\
\hline \multicolumn{2}{|l|}{ SMA/PMC } \\
\hline No. $(\%)$ of patients with values of $>0$ & $6(54.6 \%)$ \\
\hline Median (minimum, maximum) & $4.8(0.0,46.5)$ \\
\hline \multicolumn{2}{|l|}{ STN } \\
\hline No. (\%) of patients with values of $>0$ & $0(0.0 \%)$ \\
\hline Median (minimum, maximum) & $0.0(0.0,0.0)$ \\
\hline \multicolumn{2}{|l|}{ SN } \\
\hline No. $(\%)$ of patients with values of $>0$ & $8(72.7 \%)$ \\
\hline Median (minimum, maximum) & $5.8(0.0,36.3)$ \\
\hline \multicolumn{2}{|l|}{ Thalamus } \\
\hline No. (\%) of patients with values of $>0$ & $1(9.1 \%)$ \\
\hline Median (minimum, maximum) & $0.0(0.0,0.5)$ \\
\hline \multicolumn{2}{|l|}{ Outcomes } \\
\hline Preoperative UPDRS III score (range) & $42(27-55)$ \\
\hline 6-Month follow-up UPDRS III score (range) & $21(13-40)$ \\
\hline $\begin{array}{l}\text { Change in UPDRS III score (preoperative } \\
\text { minus 6-month follow-up) (range) }\end{array}$ & $15(-12-31)$ \\
\hline
\end{tabular}

Note:-PFC indicates prefrontal cortex; PPN, pedunculopontine nucleus.

${ }^{a}$ The sample median (minimum, maximum) is given for continuous variables.

PMC segment, corresponded with greater improvement in the UPDRS III score.

The globus pallidus, with the putamen, forms the lentiform nucleus and primarily exhibits inhibitory control on movement. ${ }^{28}$ The GPi has extensive connections to deep brain structures. A predominant output of the GPi consists of pallidothalamic connections comprising the ansa lenticularis and lenticular fasciculus (components of the thalamic fasciculus). The ansa lenticularis and lenticular fasciculus exit the GPi along the inferomedial and medial borders, respectively. These projections largely consist of efferent fibers to the thalamic ventralis oralis nucleus, mediodorsal nucleus, and centromedian parafascicular complex. $^{29-31}$ Additional fibers within the ansa lenticularis connect the GPi to the STN before formation of the thalamic fasciculus, which terminates in the thalamus. ${ }^{32}$ These STN-GPi connections may drive increased excitation within the GPi in PD, secondary to diminished inhibition of the STN from the GPe. ${ }^{32,33}$ Nearly $15 \%$ of GPi fibers connect to the SN, arising from the medial inferior border of the GPi. ${ }^{34}$ Connections have also been illustrated between the GPi and pedunculopontine nucleus, exiting from the medial portion of the GPi. ${ }^{35-38}$ Along the border between the GPi and GPe, inhibitory fibers exit the medial GPe and connect via the lateral border of the GPi. ${ }^{33,34}$ Last, the posterolateral border of the GPi has been shown to connect to the adjacent putamen and may produce a decrease in GPi inhibition in PD secondary to decreased putaminal excitation. ${ }^{13,39,40}$

While many of these deep connections of the GPi have been well-described, there has historically been little attention to direct cortical connections from the GPi. Tracer studies in rats and monkeys, as well as human DTI studies, have demonstrated the presence of these corticopallidal fibers, particularly in relation to the prefrontal cortex, SMA, pre-SMA, primary motor, and primary sensory cortices. ${ }^{41-45}$ Additional information regarding the connectivity of the GPi to the motor cortex (M1 region) has been elucidated from studies measuring local field potentials, where $\beta$ oscillations in the range of $13-40 \mathrm{~Hz}$ have been recorded from the GPi in a movement-related manner, similar to recordings noted in the primary motor cortex. ${ }^{46-48}$ Because the GPi is the main output structure of the basal ganglia, increased excitation of this structure produces some of the hypomotor symptoms seen in PD. However, the role of these corticopallidal connections in the pathophysiology and treatment of movement disorders has not been well-investigated to date. We hypothesized that the efficacy of DBS could be secondary to suppressing the connections arising from the M1 region.

In our study, we could elucidate direct connections between the GPi and cortex, specifically the prefrontal cortex, SMA, PMC, and $\mathrm{M} 1$ regions. As previously reported by Milardi et $\mathrm{al}^{43}$ using constrained spheric deconvolution, these fiber pathways are distinct from the hyperdirect pathway passing through the internal capsule. The results of this pilot study suggest that a larger VTA in the segment corresponding to dominant connectivity to the M1 region in patients with PD may be correlated with a greater improvement in the UPDRS III score. Findings were similar, though weaker, for the SMA/PMC segment, whereas no notable correlation with change in the UPDRS III score was observed for the VTA in the globus pallidus externus and SN segments. The idea that structural and functional connectivity measures represent independent outcome predictors has been previously shown in other treatment targets. ${ }^{8,49-51}$ For instance, Horn et al ${ }^{8}$ have shown that connectivity measures within the STN are potentially independent outcome predictors in DBS of PD. Most interesting, they found a positive correlation with the clinical response within the STN and connectivity to the SMA, while a negative correlation with the clinical response was found with the primary motor cortex. ${ }^{8}$ This anticorrelation between the DBS electrode and the primary motor cortex presenting in the STN, and not in GPi, may be explained by the development of motor adverse effects, such as verbal fluency problems, ${ }^{52}$ when stimulating the M1 region in the smaller STN.

Remarkably, in contradistinction to the prior study by da Silva et $\mathrm{al}^{13}$ we found that the posterior ventral portion of the GPi corresponds to a corticopallidal area most connected to the primary motor cortex. The area most connected to the SMA/PMC 


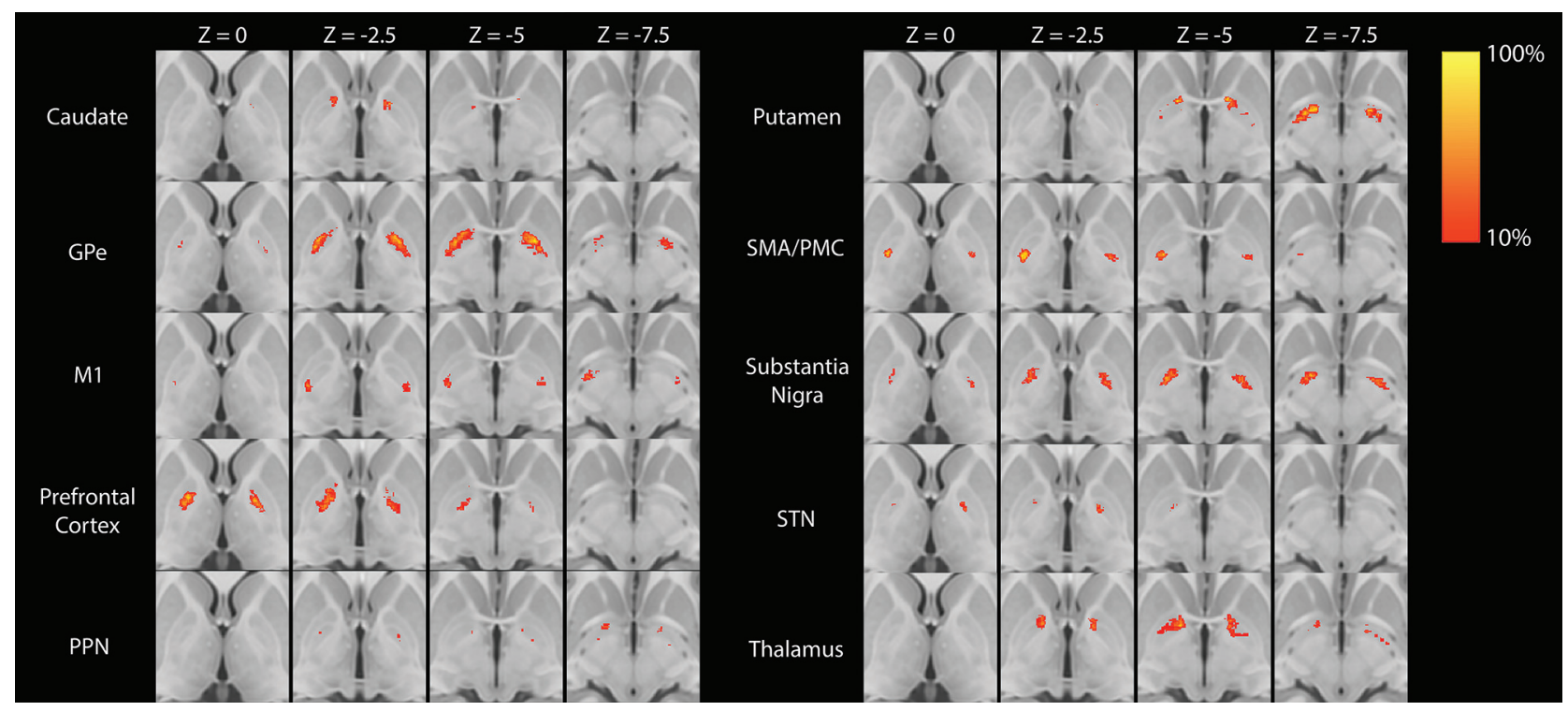

FIG 1. Statistical heat maps illustrating the group average location of each of the $10 \mathrm{GPi}$ segments. Maps are thresholded at $>10 \%$. PPN indicates pedunculopontine nucleus.
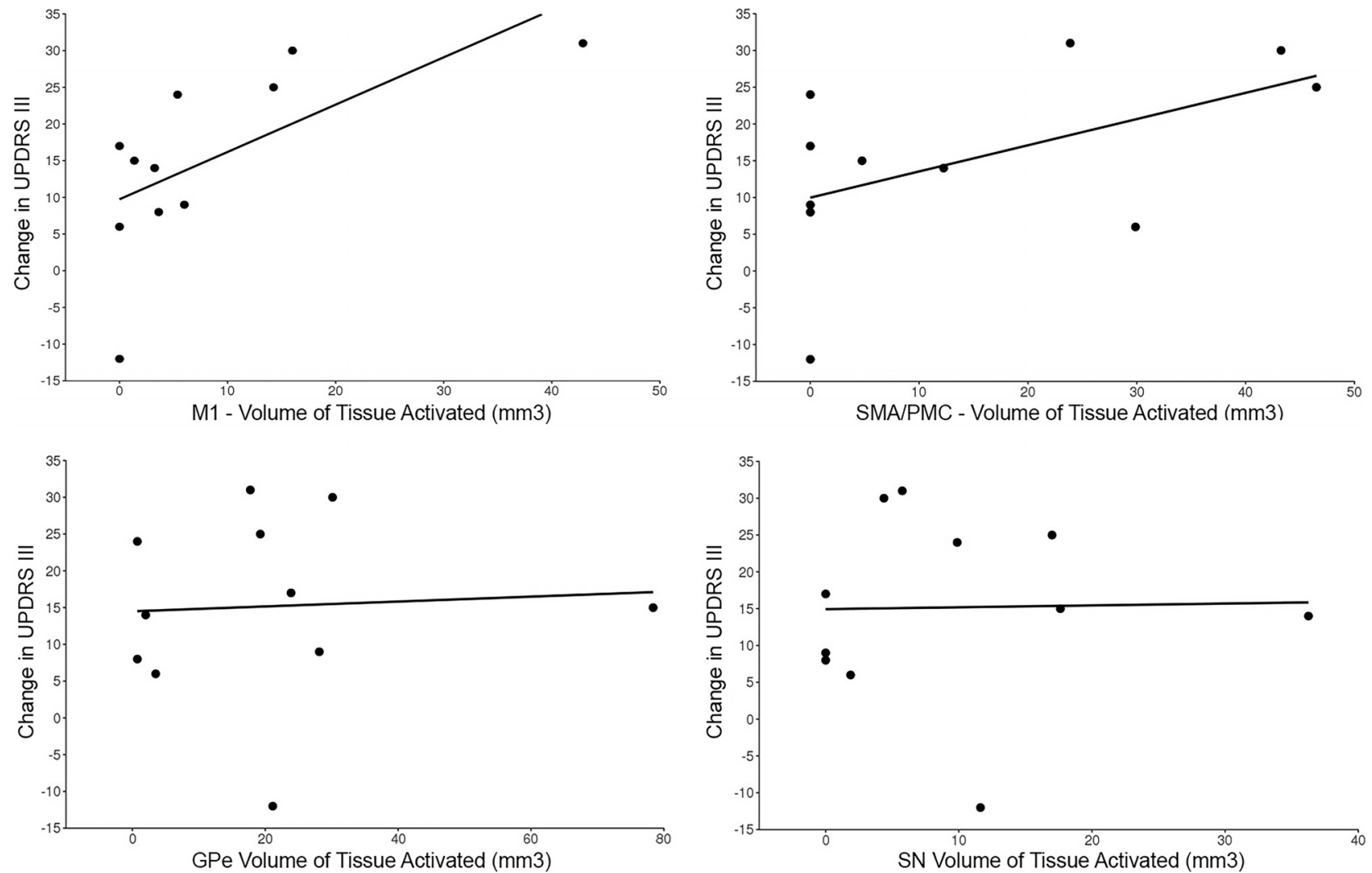

FIG 2. Scatterplots showing the change in the UPDRS score relative to volumes of tissue activated in the primary motor segment (upper left), supplementary motor area/premotor cortex segment (upper right), globus pallidus externus segment (lower left), and substantia nigra segment (bottom right).

was in proximity yet slightly more ventral in the posterior GPi (Fig 3). These findings are like those reported in prior animal tracer and DTI studies. ${ }^{41-45}$ The basis for the discrepancy with the prior study is uncertain. One potential explanation is the reduction of voxel volume by greater than a factor of 3 , potentially allowing better discrimination of these small areas of corticopallidal connectivity. ${ }^{13}$ Also, differences in the number of diffusion directions could potentially alter angular resolution, though the number of diffusion directions obtained in the prior study was not published. $^{13}$

Accuracy in structural and functional targeting is vital for successful deep brain stimulation. The GPi shows more variation in position relative to the midpoint of the line traversing the anterior/ posterior commissure line compared with other deep nuclei. This 

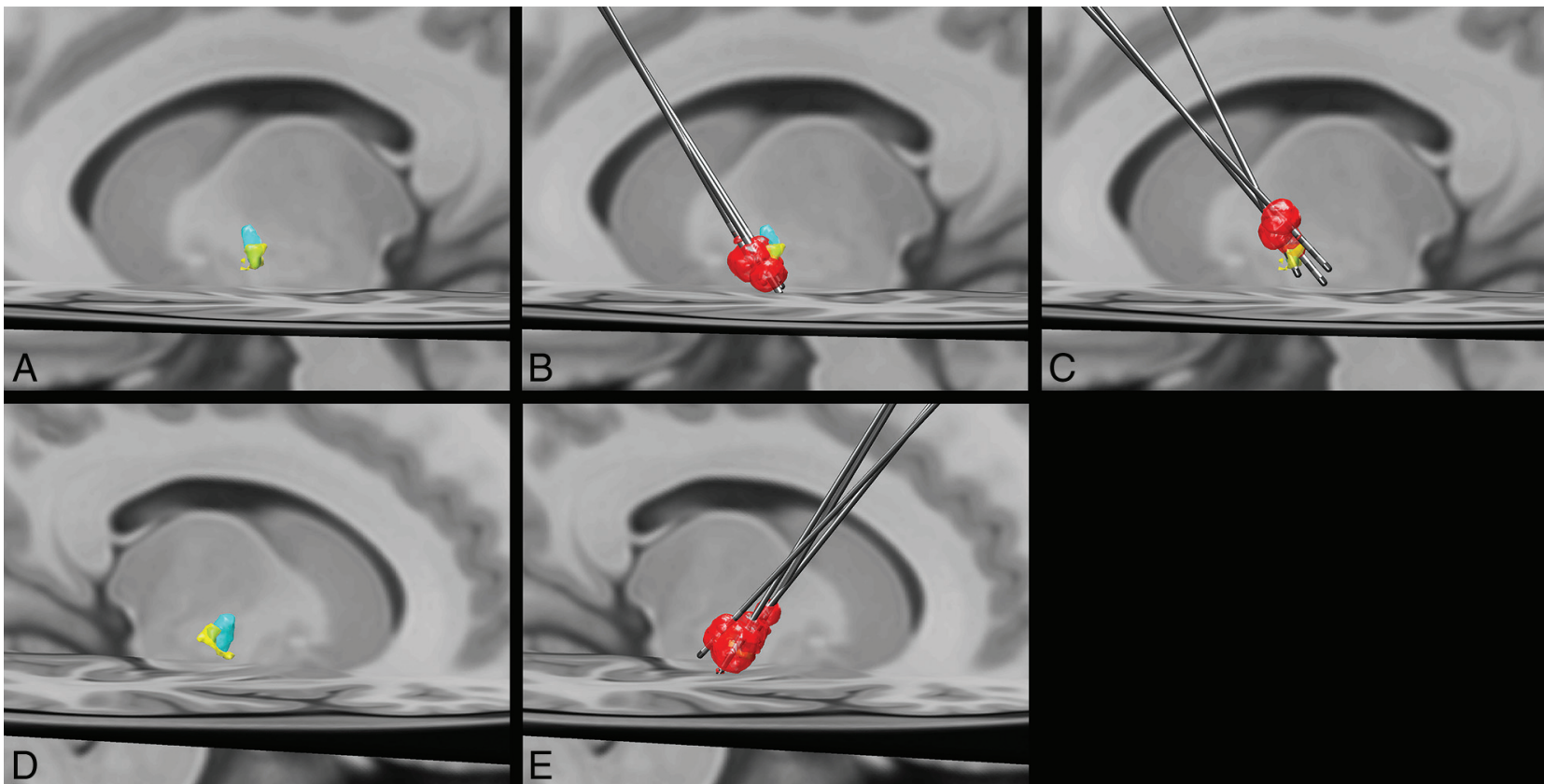

FIG 3. Left $(A)$ and right $(D)$ group average volumes for segments with maximal connectivity to the primary motor cortex (yellow) and supplementary motor area/premotor cortex (light blue). B, Left DBS leads and volumes of tissue activated (red) for the 3 patients with improvement in the UPDRS III of $>10$ (mean improvement in the score $=26.7$ ) after DBS. A more ventral location and greater overlap with the primary motor cortex segment can be appreciated. C, Left DBS leads and volumes of tissue activated (red) in the 3 patients with the least improvement in the UPDRS III score (mean improvement in the score $=0.7$ ) show a more dorsal location of the volume of tissue activated with less overlap with the primary motor cortex segment of the GPi. E, Right DBS leads and volumes of tissue activated. All patients had an improvement in the UPDRS III score of $>10$ (mean improvement in the score $=19$ ).

variation may have partly contributed to the inconsistency in outcomes of early studies of GPi-DBS. Substantial improvement in targeting by direct structural targeting, such as FGATIR MR imaging, ${ }^{4}$ and sophisticated targeting software using atlas overlays and a deformable brain atlas have contributed to improved anatomic targeting. ${ }^{4}$ The supplementation of targeting in functional neurosurgery using connectivity measures, such as DTI, is also a promising technique. ${ }^{5,6,8}$ Despite these advances, improvement in preoperative functional mapping within the deep nuclei is needed to maximize the benefits of direct-targeting methods.

While debate exists on the optimal target nucleus, 1 advantage of GPi stimulation versus STN is the size of the GPi, meaning a lower probability of off-target stimulation and unintended adverse effects. However, the need for a larger VTA to achieve a desired therapeutic effect in the GPi means increased energy usage and decreased battery life. Improvement in preoperative targeting by incorporating measures of connectivity may allow the maximization of the therapeutic effect within the GPi and help overcome some of these drawbacks by providing a more specific and focused treatment target. Given the lower rate of adverse stimulation effects, the GPi could become an even more attractive target.

Our study has several notable limitations. The greatest limitation is the small sample size, which results in a lack of precision of estimated correlations. Although we did identify 1 statistically significant association despite the small sample size, larger studies are needed to better elucidate the role of our methods in supplementing preoperative GPi targeting. However, the small sample size is appropriate for the pilot nature of the study. Second, the retrospective nature only allows modeling of VTAs from data that were historically recorded, limiting comparison of different stimulation parameters in each patient. Third, the role of corticopallidal connections in PD pathophysiology warrants further exploration, given the paucity of existing knowledge. Because these connections in humans have largely been described by noninvasive MR tractography, further confirmation of their existence in anatomic specimens is needed. Last, the methods used in this study inherently oversimplify the connectivity profiles of the VTA because each voxel is assigned to only the target of the most propagated paths. While this feature potentially limits the methods in understanding the pathophysiology behind GPi-DBS, the goal of this investigation was the establishment of a reliable and reproducible subject-specific functional preoperative target for GPiDBS placement.

\section{CONCLUSIONS}

Despite advances in preoperative targeting for DBS, reproducible methods for defining subject-specific connectivity targets with the GPi are lacking. Our preliminary study has shown an independent predictive value of connectivity-based segmentation of the GPi on patient outcomes. Our clinically feasible technique may be of benefit in supplementing direct structural targeting, as well as in DBS programming, to maximize therapeutic outcomes. Larger studies will be needed to further corroborate these findings.

Disclosures: Erik H. Middlebrooks—UNRELATED: Grant: Varian Medical Systems; Consultancy: Varian Medical Systems. Kelly D. Foote-UNRELATED: Consultancy: Medtronic; Grants/Grants Pending: Medtronic, Boston Scientific, St. Jude.* Michael S. Okun-UNRELATED: Grant: National Institutes of Health, Comments: DBS re- 
search is supported by R01 NR014852 and R01NS096008.* *Money paid to the institution.

\section{REFERENCES}

1. Obeso JA, Olanow CW, Rodriguez-Oroz MC, et al; Deep-Brain Stimulation for Parkinson's Disease Study Group. Deep-brain stimulation of the subthalamic nucleus or the pars interna of the globus pallidus in Parkinson's disease. N Engl J Med 2001;345:956-63 CrossRef Medline

2. Okun MS, Fernandez HH, Wu SS, et al. Cognition and mood in Parkinson's disease in subthalamic nucleus versus globus pallidus interna deep brain stimulation: the COMPARE trial. Ann Neurol 2009;65:586-95 CrossRef Medline

3. Odekerken VJ, van Laar T, Staal MJ, et al. Subthalamic nucleus versus globus pallidus bilateral deep brain stimulation for advanced Parkinson's disease (NSTAPS study): a randomised controlled trial. Lancet Neurol 2013;12:37-44 CrossRef Medline

4. Sudhyadhom A, Haq IU, Foote KD, et al. A high resolution and high contrast MRI for differentiation of subcortical structures for DBS targeting: the Fast Gray Matter Acquisition T1 Inversion Recovery (FGATIR). Neuroimage 2009;47(Suppl 2):T44-52 CrossRef Medline

5. Alho AT, Hamani C, Alho EJ, et al. Magnetic resonance diffusion tensor imaging for the pedunculopontine nucleus: proof of concept and histological correlation. Brain Struct Funct 2017;222:2547-58 CrossRef Medline

6. Sajonz BE, Amtage F, Reinacher PC, et al. Deep Brain Stimulation for Tremor Tractographic Versus Traditional (DISTINCT): study protocol of a randomized controlled feasibility trial. JMIR Res Protoc 2016;5:e244 CrossRef Medline

7. Pouratian N, Zheng Z, Bari AA, et al. Multi-institutional evaluation of deep brain stimulation targeting using probabilistic connectivity-based thalamic segmentation. J Neurosurg 2011;115:995-1004 CrossRef Medline

8. Horn A, Reich M, Vorwerk J, et al. Connectivity predicts deep brain stimulation outcome in Parkinson disease. Ann Neurol 2017;82: 67-78 CrossRef Medline

9. Behrens TE, Berg HJ, Jbabdi S, et al. Probabilistic diffusion tractography with multiple fibre orientations: what can we gain? Neuroimage 2007;34:144-55 CrossRef Medline

10. Behrens TE, Johansen-Berg H, Woolrich MW, et al. Non-invasive mapping of connections between human thalamus and cortex using diffusion imaging. Nat Neurosci 2003;6:750-57 CrossRef Medline

11. Traynor C, Heckemann RA, Hammers A, et al. Reproducibility of thalamic segmentation based on probabilistic tractography. Neuroimage 2010;52:69-85 CrossRef Medline

12. Elias WJ, Zheng ZA, Domer P, et al. Validation of connectivity-based thalamic segmentation with direct electrophysiologic recordings from human sensory thalamus. Neuroimage 2012;59:2025-34 CrossRef Medline

13. da Silva NM, Ahmadi SA, Tafula SN, et al. A diffusion-based connectivity map of the GPi for optimised stereotactic targeting in DBS. Neuroimage 2017;144:83-91 CrossRef Medline

14. Parent A, Hazrati LN. Functional anatomy of the basal ganglia, I: the cortico-basal ganglia-thalamo-cortical loop. Brain Res Brain Res Rev 1995;20:91-127 CrossRef Medline

15. Tisch S, Zrinzo L, Limousin P, et al. Effect of electrode contact location on clinical efficacy of pallidal deep brain stimulation in primary generalised dystonia. J Neurol Neurosurg Psychiatry 2007;78: 1314-19 CrossRef Medline

16. Prodoehl J, Yu H, Little DM, et al. Region of interest template for the human basal ganglia: comparing EPI and standardized space approaches. Neuroimage 2008;39:956-65 CrossRef Medline

17. Keuken MC, Forstmann BU. A probabilistic atlas of the basal ganglia using 7 T MRI. Data Brief 2015;4:577-82 CrossRef Medline

18. Mayka MA, Corcos DM, Leurgans SE, et al. Three-dimensional locations and boundaries of motor and premotor cortices as defined by functional brain imaging: a meta-analysis. Neuroimage 2006;31: 1453-74 CrossRef Medline

19. Makris N, Goldstein JM, Kennedy D, et al. Decreased volume of left and total anterior insular lobule in schizophrenia. Schizophr Res 2006;83:155-71 CrossRef Medline

20. Frazier JA, Chiu S, Breeze JL, et al. Structural brain magnetic resonance imaging of limbic and thalamic volumes in pediatric bipolar disorder. Am J Psychiatry 2005;162:1256-65 CrossRef Medline

21. Desikan RS, Ségonne F, Fischl B, et al. An automated labeling system for subdividing the human cerebral cortex on MRI scans into gyral based regions of interest. Neuroimage 2006;31:968-80 CrossRef Medline

22. Goldstein JM, Seidman LJ, Makris N, et al. Hypothalamic abnormalities in schizophrenia: sex effects and genetic vulnerability. Biol Psychiatry 2007;61:935-45 Medline

23. Avants BB, Epstein CL, Grossman M, et al. Symmetric diffeomorphic image registration with cross-correlation: evaluating automated labeling of elderly and neurodegenerative brain. Med Image Anal 2008;12:26-41 CrossRef Medline

24. Fonov V, Evans AC, Botteron K, et al; Brain Development Cooperative Group. Unbiased average age-appropriate atlases for pediatric studies. Neuroimage 2011;54:313-27 CrossRef Medline

25. Horn A, Kühn AA. Lead-DBS: a toolbox for deep brain stimulation electrode localizations and visualizations. Neuroimage 2015;107: 127-35 CrossRef Medline

26. Wichmann T, Dostrovsky JO. Pathological basal ganglia activity in movement disorders. Neuroscience 2011;198:232-44 CrossRef Medline

27. McCairn KW, Iriki A, Isoda M. Common therapeutic mechanisms of pallidal deep brain stimulation for hypo- and hyperkinetic movement disorders. J Neurophysiol 2015;114:2090-104 CrossRef Medline

28. Yagmurlu K, Vlasak AL, Rhoton AL Jr. Three-dimensional topographic fiber tract anatomy of the cerebrum. Neurosurgery 2015; 11(Suppl 2):274-305; discussion 305 CrossRef Medline

29. Baron MS, Sidibé M, DeLong MR, et al. Course of motor and associative pallidothalamic projections in monkeys. J Comp Neurol 2001;429:490-501 Medline

30. Severin CM, Young PA, Massapust LC. Pallidothalamic projections in the rat. J Comp Neurol 1976;166:491-502 CrossRef Medline

31. Gallay MN, Jeanmonod D, Liu J, et al. Human pallidothalamic and cerebellothalamic tracts: anatomical basis for functional stereotactic neurosurgery. Brain Struct Funct 2008;212:443-63 CrossRef Medline

32. Hashimoto T, Elder CM, Okun MS, et al. Stimulation of the subthalamic nucleus changes the firing pattern of pallidal neurons. $J \mathrm{Neu}$ rosci 2003;23:1916-23 Medline

33. Delong MR, Georgopoulos AP, Crutcher MD, et al. Functional organization of the basal ganglia: contributions of single-cell recording studies. Ciba Found Symp 1984;107:64-82 Medline

34. Plantinga BR, Roebroeck A, Kemper VG, et al. Ultra-high field MRI post mortem structural connectivity of the human subthalamic nucleus, substantia nigra, and globus pallidus. Front Neuroanat 2016 ; 10:66 CrossRef Medline

35. Perez-Costas E, Melendez-Ferro M, Roberts RC. Basal ganglia pathology in schizophrenia: dopamine connections and anomalies. J Neurochem 2010;113:287-302 CrossRef Medline

36. Martinez-Gonzalez C, Bolam JP, Mena-Segovia J. Topographical organization of the pedunculopontine nucleus. Front Neuroanat 2011; 5:22 CrossRef Medline

37. Shink E, Sidibé M, Smith Y. Efferent connections of the internal globus pallidus in the squirrel monkey, II: topography and synaptic organization of pallidal efferents to the pedunculopontine nucleus. J Comp Neurol 1997;382:348-63 Medline

38. Zhang J, Wang ZI, Baker KB, et al. Effect of globus pallidus internus stimulation on neuronal activity in the pedunculopontine tegmental nucleus in the primate model of Parkinson's disease. Exp Neurol 2012;233:575-80 CrossRef Medline

39. Xiao Y, Fonov V, Chakravarty MM, et al. A dataset of multi-contrast 
population-averaged brain MRI atlases of a Parkinson's disease cohort. Data Brief 2017;12:370-79 CrossRef Medline

40. Detante O, Vercueil L, Thobois S, et al. Globus pallidus internus stimulation in primary generalized dystonia: a H215O PET study. Brain 2004;127:1899-908 CrossRef Medline

41. Naito A, Kita H. The cortico-pallidal projection in the rat: an anterograde tracing study with biotinylated dextran amine. Brain Res 1994;653:251-57 CrossRef Medline

42. Rozanski VE, Vollmar C, Cunha JP, et al. Connectivity patterns of pallidal DBS electrodes in focal dystonia: a diffusion tensor tractography study. Neuroimage 2014;84:435-42 CrossRef Medline

43. Milardi D, Gaeta M, Marino S, et al. Basal ganglia network by constrained spherical deconvolution: a possible cortico-pallidal pathway? Mov Disord 2015;30:342-49 CrossRef Medline

44. Akkal D, Dum RP, Strick PL. Supplementary motor area and presupplementary motor area: targets of basal ganglia and cerebellar output. J Neurosci 2007;27:10659-73 CrossRef Medline

45. Hoover JE, Strick PL. Multiple output channels in the basal ganglia. Science 1993;259:819-21 CrossRef Medline

46. Brücke C, Kempf F, Kupsch A, et al. Movement-related synchronization of gamma activity is lateralized in patients with dystonia. Eur J Neurosci 2008;27:2322-29 CrossRef Medline
47. Tsang EW, Hamani C, Moro E, et al. Movement related potentials and oscillatory activities in the human internal globus pallidus during voluntary movements. J Neurol Neurosurg Psychiatry 2012;83: 91-97 CrossRef Medline

48. Talakoub O, Neagu B, Udupa K, et al. Time-course of coherence in the human basal ganglia during voluntary movements. Sci Rep 2016;6:34930 CrossRef Medline

49. Brunenberg EJL, Moeskops $P$, Backes WH, et al. Structural and resting state functional connectivity of the subthalamic nucleus: identification of motor STN parts and the hyperdirect pathway. PLoS One 2012;7:e39061 CrossRef Medline

50. Lambert C, Zrinzo L, Nagy Z, et al. Confirmation of functional zones within the human subthalamic nucleus: patterns of connectivity and sub-parcellation using diffusion weighted imaging. Neuroimage 2012;60:83-94 CrossRef Medline

51. Vanegas-Arroyave N, Lauro PM, Huang L, et al. Tractography patterns of subthalamic nucleus deep brain stimulation. Brain 2016; 139:1200-10 CrossRef Medline

52. Dietz J, Noecker AM, McIntyre CC, et al. Stimulation region within the globus pallidus does not affect verbal fluency performance. Brain Stimul 2013;6:248-53 CrossRef Medline 